\title{
Biocomposite Interference Screws in Anterior Cruciate Ligament Reconstruction: Osteoconductivity and Degradation
}

\author{
F. Alan Barber, M.D., and W. D. Dockery, M.D.
}

\begin{abstract}
Purpose: To evaluate the long-term in vivo degradation of an amorphous stereoisomer combined with micro $\beta$-tricalcium phosphate poly levo $(96 \%) /$ dextro $(4 \%)$ lactide beta-tricalcium phosphate biocomposite interference screw. Methods: A study approved by the institutional review board of in vivo biologic behavior of the screw was initiated in 2011 using an anterior cruciate ligament (ACL) reconstruction model. Twenty patients undergoing bone-patellar tendon-bone ACL reconstruction fixed at the femur and tibia with these biocomposite screws followed at least 36 months were evaluated by physical, radiographic, and computed tomography (CT) evaluations. Lysholm, Tegner, Cincinnati, and International Knee Documentation Committee scores were obtained. CT Hounsfield unit (HU) data were obtained at the femoral and tibial screw and other bone sites. An ossification quality score (range 1-4) was used to determine osteoconductivity at the screw sites. Results: In total, 11 male and 9 female patients evaluated by CT scan and radiographs a mean of 41 months postsurgery (range, 37-51) showed bone plug healing to the tunnel wall and the screw replaced with calcified and nontrabecular material. Osteoconductivity was present in 34 of 40 tunnels (85\%) and nearly complete or complete (type 3 or 4 ossification) in 10 of 40 (25\%). Mean screw-site densities (femoral 239 HU; tibial 290 HU) were consistent with cancellous bone density. One positive pivot-shift test was found. Lysholm, Cincinnati, Tegner, and International Knee Documentation Committee activity scores improved from 46.9, 43.5, 1.9, and 1.7 preoperatively to 92, 90.2, 6.0, and 3.2 at follow-up, respectively. The average postoperative Single Assessment Numeric Evaluation score was 86 and mean KT-1000 arthrometer difference was $0.32 \mathrm{~mm}$. Conclusions: The micro $\beta$-tricalcium phosphate poly levo $(96 \%)$ /dextro $(4 \%)$ lactide beta-tricalcium phosphate interference screw was replaced by calcified, nontrabecular material a mean of 42 months after implantation in a bone-patellar tendon-bone ACL reconstruction model. Osteoconductivity was confirmed. Level of Evidence: Level IV (therapeutic case series).
\end{abstract}

B iodegradable interference screw fixation for anterior cruciate ligament (ACL) reconstruction was first reported in 1995. ${ }^{1}$ Different biodegradable materials have been introduced since the first poly L-lactide (PLLA) implants. The recent addition of calcium bioceramics to biodegradable polymers creates a biocomposite material. These biocomposite materials can be used in interference screws with improved ossification,

From Plano Orthopedic and Sports Medicine Center, Plano (F.A.B.); and American Radiology Associates P.A., Dallas (W.D.D.), Texas, U.S.A.

The authors report the following potential conflicts of interest or sources of funding: This study was supported by funding or grants from the F. Alan Barber M.D., F.A.C.S., Research Fund and ConMed Linvatec. One or more of the researchers or an affiliated institute (F.A.B.) has received (or agreed to receive) from a commercial entity something of value (exceeding the equivalent of US\$500) related to this research. Both ConMed Linvatec and the F. Alan Barber, M.D., F.A.C.S. Research Fund covered the cost of the magnetic resonance imaging and institutional review board fees. F.A.B. receives payment for lectures including service on speakers bureaus from DePuy Mitek; and receives payment for patents (planned, pending or issued) from DePuy Mitek. reduced tunnel widening, and increased cortical bone formation. ${ }^{2}$ Since their introduction, biocomposite interference screw use has increased $7 \%$ per year. ${ }^{3}$ The eventual biologic outcome of different biocomposite implants is important since different isomers of PLLA, copolymers of PLLA and polyglycolic acid, and different calcium-containing compounds are used. Previous clinical reports have confirmed that devices made from

\footnotetext{
Full ICMJE author disclosure forms are available for this article online, as supplementary material.

Received September 16, 2019; accepted October 7, 2019.

Address correspondence to F. Alan Barber, M.D., Plano Orthopedic and Sports Medicine Center, 5228 West Plano Parkway, Plano, TX 75093, U.S.A.

(C) 2019 THE AUTHORS. Published by Elsevier Inc. on behalf of the Arthroscopy Association of North America. This is an open access article under the CC BY-NC-ND license (http://creativecommons.org/licenses/by-nc-nd/4.0/). 2666-061X/191129

https://doi.org/10.1016/j.asmr.2019.10.001
} 


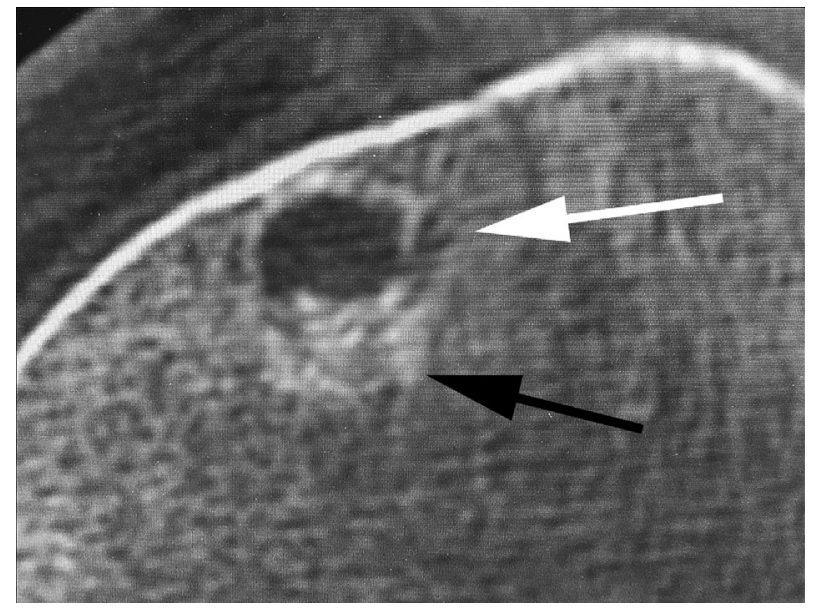

Fig 1. Type 1 ossification has little or no ossification at the previous screw site (white arrow) and is located adjacent to the bone plug, which has healed to the adjacent tunnel wall (black arrow). (Copyright by F. Alan Barber, M.D., F.A.C.S.)

biocomposites of PLLA and beta-tricalcium phosphate ( $\beta$-TCP) completely degrade over time and demonstrate osteoconductivity (the growth of bony tissue into the structure of an implant). ${ }^{4-8}$ No implant without a bioceramic has demonstrated any evidence of osteoconductivity. 9,10

Adverse events also have been reported with biocomposite implants. The poly D,L-lactide-co-glycolide $(65 \%) / \mathrm{CaCO}_{3}(35 \%)$ screw was withdrawn from the market because it demonstrated a very rapid degradation with marked inflammatory responses. ${ }^{11,12}$ Another biocomposite made from poly-L (70)/D (30) lactide (LA) and $\beta$-TCP was associated with subcutaneous pseudocyst formation. ${ }^{13}$ The accurate assessment of the degradation and osteoconductive behavior of an implant is important and CT scanning rather than magnetic resonance imaging (MRI) assessment is the established methodology for this task. ${ }^{14-16}$ The purpose of this study is to evaluate the long-term in vivo degradation of an amorphous stereoisomer (poly levo [96\%]/dextro [4\%] lactide) combined with micro $\beta$-TCP (i.e., PL [96]/D [4] LA/ $\beta$-TCP) biocomposite interference screw. Our hypothesis was that an amorphous stereoisomer (PL [96\%]/D [4\%] LA) combined with micro $\beta$-TCP would demonstrate significant osteoconductivity.

\section{Methods}

With institutional review board approval, a prospective in vivo evaluation of a PL (96)/D (4) LA/ $\beta$-TCP interference screw (Genesys-Matryx screw; ConMed Linvatec, Largo, FL) was initiated in August 2011 using a consecutive series of patients undergoing bone-patella tendon-bone ACL autograft or allograft reconstruction. Starting no earlier than 3 years after the index surgery, computed tomography (CT) scans of the knees were obtained to evaluate the behavior of the interference screw in bone.

Twenty included patients underwent ACL reconstructions with the biocomposite PL (96)/D (4) LA/ $\beta$-TCP screw. All patients were evaluated preoperatively with physical and radiographic examinations and clinical outcome measures: Lysholm, Cincinnati, Tegner, and International Knee Documentation Committee (IKDC) activity scores. Those enrolled were evaluated at follow-up using Lysholm, Cincinnati, Tegner, and IKDC activity scores, physical examination, KT-1000 arthrometer (Medmetric, San Diego, CA) testing, radiographic, and CT scans. Postoperative Single Assessment Numeric Evaluation scores were also obtained. ${ }^{17}$ Standard knee radiographs included standing posteroanterior, Merchant, tunnel notch, and true lateral views.

Included in this study were bone-patellar tendon-bone ACL reconstructions with interference screw fixation at both the femur and the tibia using the biocomposite interference screw. All patients were followed a minimum of 36 months after surgery. ACL revision procedures were allowed. Exclusion criteria were bilateral knee or multiple-ligament reconstructions, previous bone procedures in the area of the ACL graft attachments, metabolic bone disease, fractures, or tumors that involved the knee.

The CT scan obtained evaluated screw degradation and absorption and looked for evidence of bone ingrowth into the area of the degraded screw. The technique used followed the established protocol of Barber and Dockery. ${ }^{4-7,9}$ A CT scan was used instead of MRI because it provides an objective, qualitative, and

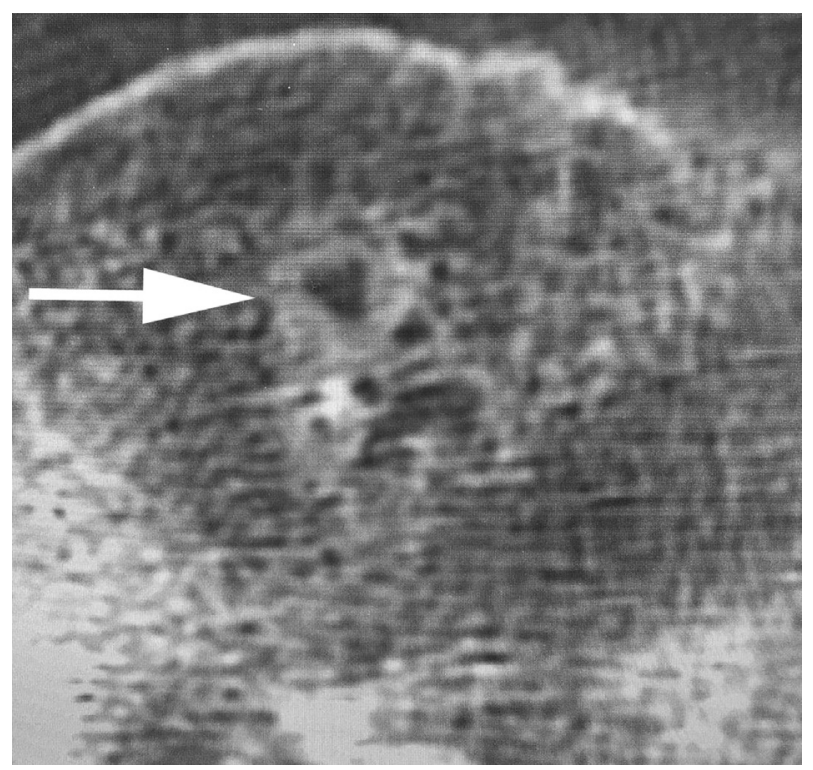

Fig 2. Screw sites with type 2 ossification (arrow) have some ossification at the previous screw location that is discontinuous. (Copyright by F. Alan Barber, M.D., F.A.C.S.) 


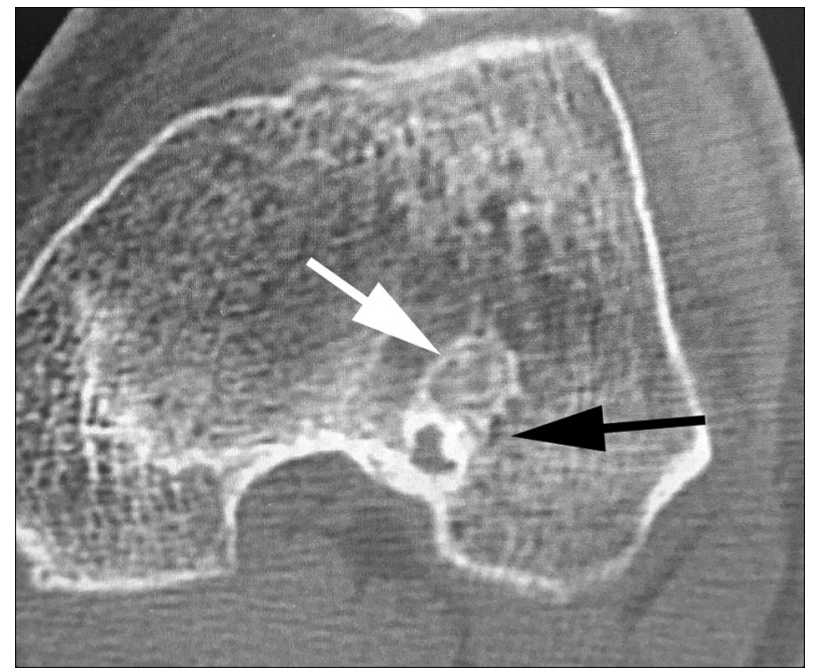

Fig 3. In type 3 ossification, the previous screw site (white arrow) is filled with material similar in density to the surrounding cancellous bone but has a thin sclerotic rim. The previous bone plug is well attached to the previous tunnel wall (black arrow). (Copyright by F. Alan Barber, M.D., F.A.C.S.)

quantitative assessment in Hounsfield units (HU) of the material at the previous implant sites. Multiplanar sagittal and coronal images were reviewed by an experienced radiologist (W.D.D.) not involved in the surgical procedure. The institutional review board restricted the study to 20 patients because of the issue of radiation exposure and to be consistent with previous published studies.

A Barber-Dockery ossification quality score was assigned to each interference screw site. ${ }^{4}$ This ossification quality score has 4 levels. Type 1 ossification shows little or no ossification beyond an obviously threaded screw tract filled with soft-tissue density (Fig 1). Type 2 ossification shows some ossification that is discontinuous or with a wide lucent rim (Fig 2). Type 3 ossification fills most of the screw site and has a thin lucent rim (Fig 3), and type 4 has good, complete ossification with a vague tract border, suggesting good incorporation with the adjacent cancellous bone (Fig 4).

In addition, the HU scores for all screw and bone plug sites were recorded. These sites were readily identifiable because the ACL graft bone plugs were easily located and the interference screws were always located immediately adjacent to the bone plugs. Areas of obvious focal fat or focal sclerosis were avoided for the HU data collection. The distance between the tibial screw site and the tibial joint line varied because of variations in the patella tendon length. Different axial slices were used to obtain the femoral and tibial measurements because the slices were not in line with the respective tunnel angles.

The primary endpoint was the reabsorption status of the biocomposite interference screw. The secondary endpoint was whether osteoconductivity and bone ingrowth was apparent in the area previously occupied by the biocomposite interference screw. The third endpoint was the clinical outcomes of those patients evaluated.

\section{Surgical Technique}

All patients underwent an arthroscopically assisted ACL reconstruction with a patellar tendon graft performed by a single surgeon (F.A.B.). Starting in February 2012, the transtibial femoral tunnel technique was changed to an accessory anterior medial portal technique. Aperture fixation of the bone plug at the femoral tunnel outlet was achieved in all cases. The femoral tunnels were $9 \mathrm{~mm}$ in diameter, as was the prepared bone plug inserted into the femoral tunnel. The tibial bone plugs and tibial insertion tunnels also were matched and $10 \mathrm{~mm}$ in diameter. Both femoral and tibial interference screws measured $8 \mathrm{~mm}$ by 20 $\mathrm{mm}$. These cannulated screws were inserted over a guidewire, facilitating accurate screw placement and avoiding divergence. Femoral side preinsertion tapping was performed.

\section{Statistical Analysis}

A Student $t$ test determined differences between preoperative and postoperative scores. Statistical significance level was $P<.05$.

\section{Results}

A total of 20 patients were examined at an average of 42 months postsurgery (range 37-52). The average age was 33.4 (range 15-49). There were 11 male and 9 female patients, and the right leg was reconstructed in 7 and the left in 13.

Clinical outcome measures improved from the preoperative state in all patients. The preoperative and

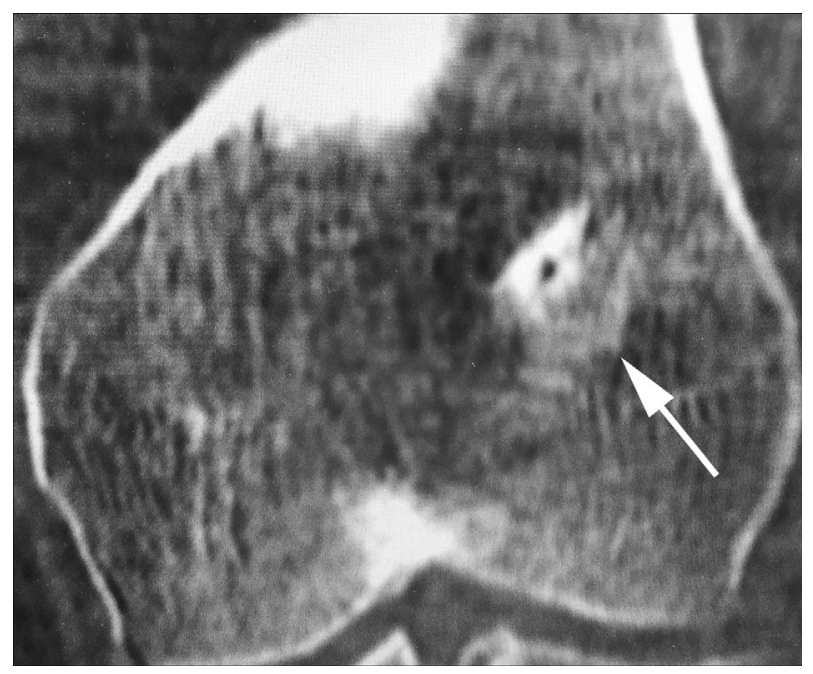

Fig 4. Type 4 ossification (arrow) demonstrates complete filling of the previous screw site with an indistinct border and no sclerotic rim. (Copyright by F. Alan Barber, M.D., F.A.C.S.) 
Table 1. Osteoconductivity Assessed With Established Ossification Quality Score

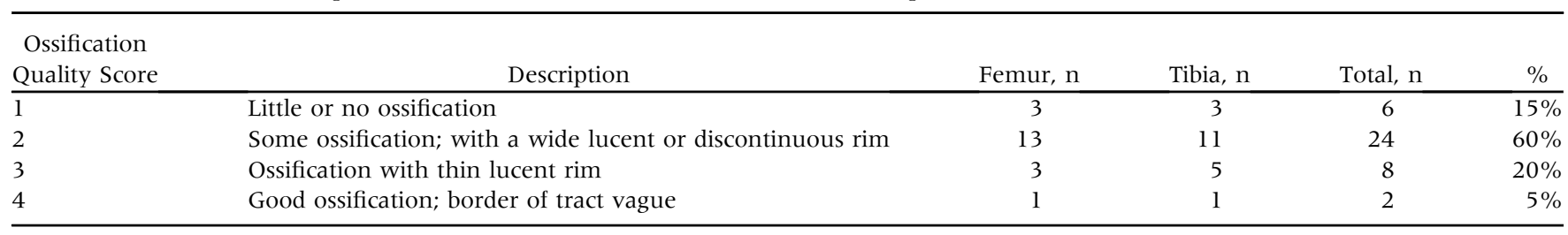

postoperative scores were as follows: Lysholm $46.9 \pm$ 20.4 versus $92 \pm 12.6$; Cincinnati $43.5 \pm 18.1$ versus $90.2 \pm 15$; Tegner $1.9 \pm 0.68$ versus $6.0 \pm 2.1$; and IKDC activity score $1.7 \pm 0.88$ versus $3.2 \pm 1.4$. The average postoperative Single Assessment Numeric Evaluation score was 86.2 \pm 12.4 (range 50-100).

On physical examination, all patients demonstrated positive Lachman and pivot shift tests preoperatively. Postoperatively at an average of 42 months postsurgery, there was only one patient with a positive Lachman test. One positive pivot shift test was noted in the same patient. ACL revision was not required in either this patient or any others in the cohort. The mean manual maximum KT-1000 score was $0.32 \mathrm{~mm}$, with none exceeding $3 \mathrm{~mm}$. Otherwise, no intraoperative or postoperative complications were encountered in these patients during the study period.

Radiographs showed that the bone plugs were incorporated into the tunnel walls and the biocomposite screws sites showed no tunnel widening, lytic, or cystic changes. The secondary endpoint was the osteoconductivity and bone ingrowth status at the previous screw site. Grading using the Barber-Dockery ossification score (Table 1$)^{4}$ demonstrated that osteoconductivity was found in 34 of 40 tunnels $(85 \%)$ and that 10 of $40(25 \%)$ of these screw sites had nearly complete or complete ossification.

The CT scans showed that the bone plugs had healed to the adjacent tunnel wall and were significantly denser than the adjacent screw sites $(P<.0001$ for the tibial sites and $P<.0001$ for the femoral sites). The mean tibial and femoral screw site densities (239 HU and $290 \mathrm{HU}$ respectively) were not statistically different from the adjacent cancellous bone $(P=.18)$ (Table 2). The screws were replaced with material that was calcified and nontrabecular. The graft tunnels showed

Table 2. Computed Tomography Scan Density for Various Locations Studied

\begin{tabular}{lcc}
\hline \multicolumn{1}{c}{ Location } & Mean \pm SD, HU & Range, HU \\
\hline Femoral screw site & $239 \pm 37$ & $161-297$ \\
Femoral bone plug & $708 \pm 200$ & $432-1140$ \\
Tibial screw site & $290 \pm 32$ & $229-348$ \\
Tibial bone plug & $568 \pm 180$ & $253-857$ \\
Cancellous bone & $242 \pm 71$ & $151-417$ \\
Muscle & $62 \pm 7.4$ & $48-76$ \\
\hline
\end{tabular}

HU, Hounsfield units; SD, standard deviation. signs of screw tracts with some threaded margins. A thin rim of low-density material was observed at the screw sites with lower ossification scores. The material replacing these screws was not trabeculated although some cases with higher ossification scores were clearly calcified and resembled trabeculated bone (Figures 3 and 4 ). The HU densities were determined and are reported in Table 2.

\section{Discussion}

The biocomposite interference fixation screw combining micro $\beta$-TCP with a $96 \%-4 \%$ stereoisomer of levo and dextro lactide completely degraded at a mean interval of 41 months and displayed osteoconductivity in $85 \%$ of the screw sites. Based on the ossification score, this osteoconductivity resulted in complete or nearly complete bone ingrowth in $25 \%$ of the screw sites. Significant improvement was documented from the preoperative status in Lysholm, Cincinnati, Tegner, and IKDC activity scores.

Previous CT scan studies report complete or nearly complete osteoconductivity in between $33 \%{ }^{4}$ to $50 \%{ }^{5}$ of the implant sites. Depending on the nature of the principal biodegradable polymer (a stereoisomer or copolymer) and possibly the associated calcium containing material, these biocomposite interference screws completely degrade over a range of time, starting as early as 36 months, but can be much longer. Selfreinforcement of the polymer material does not seem to adversely affect the material performance. ${ }^{8}$ In fact, the current study screw had the same composition as another study ${ }^{8}$ but without self-reinforcement and did not demonstrate the same osteoconductivity. In addition, an overly amorphous biocomposite, such as was used in the poly D,L-lactide-co-glycolide $(65 \%) / \mathrm{CaCO}_{3}$ $(35 \%)$ screw, can result in marked inflammatory reactions. ${ }^{11,18,19}$ The poly D,L-lactide-co-glycolide $(65 \%)$ / $\mathrm{CaCO}_{3}(35 \%)$ screw clinical experience was characterized by osteolysis, tunnel widening, and cystic formation in $88 \%$ of cases on MRI at 1 year. ${ }^{1,18,19}$ The biocomposite screw that is the subject of the current report demonstrated osteoconductivity.

The most commonly used bioceramic in biocomposite implants is $\beta$-TCP. The current device investigated contains $23 \% \beta$-TCP. This is a lower concentration than other biocomposite screws studied. However, the Mega Fix biocomposite (Karl Storz, Tuttlingen, Germany) 
device reportedly has $20 \% \beta$-TCP. Although there is no established "best concentration" of $\beta$-TCP, a greater concentration seems to be linked to more osteoconductive behavior. ${ }^{5}$

The $\beta$-TCP concentration can affect the absorption rate and osteoconductive behavior. Ntagiopoulos et al. ${ }^{7}$ studied the effect of different concentrations of $\beta$-TCP using 2 different interference screws. Biocomposite interference screws containing either $30 \% \beta$-TCP or $60 \% \beta$-TCP were evaluated using CT scans between 29 and 45 months' post-ACL reconstruction. ${ }^{7}$ Osteoconductive behavior was present in $97 \%$ of the $30 \%$ $\beta$-TCP and $96 \%$ of the $60 \% \beta$-TCP screws. The $30 \%$ $\beta$-TCP biocomposite screws demonstrated nearly complete or complete ossification (Barber-Dockery grades 3 and 4 ) in $93 \%$ of the screw sites, whereas the $60 \%$ $\beta$-TCP screws demonstrated nearly complete or complete ossification (grades 3 and 4 ) in only $60 \%{ }^{7}$ The greater $\beta$-TCP concentration created a more amorphous polymer and resulted in the screw absorbing more rapidly. The authors' explanation for this observed phenomenon was that the $30 \% \beta$-TCP screws were used to secure bone plugs and therefore were surrounded by bone in contrast to the $60 \% \beta$-TCP screws which were used to fix hamstrings autografts. An alternate theory is that the greater $\beta$-TCP concentration released more phosphate base into the surrounding environment during degradation resulting in a greater $\mathrm{pH}$, which inhibited osteoconductive behavior. It is also possible that the more amorphous implant rapidly degraded in an increased inflammatory reaction occurred.

A different biocomposite screw also containing 30\% $\beta$-TCP but combined with a copolymer of PLLA/polyglycolic acid $(70 \%)$ performed similarly by demonstrating osteoconductivity in $81 \%$ of the screw sites with complete or nearly complete filling in $50 \% .^{5,10}$ The same material used for suture anchors also demonstrated nearly complete or complete ossification (Barber-Dockery grades 3 and 4$)$ at $50 \%$ of the anchor sites. ${ }^{6}$

Hydroxyapatite is another bioceramic that has been combined with PLLA to create a biocomposite material. A PLLA $(75 \%) / \mathrm{HA}(25 \%)$ interference screw was studied after ACL reconstruction for osteoconductive behavior. ${ }^{20}$ All the biocomposite screws completely degraded at 5 years with the ossification scores, improving over time. At the 5-year interval, $90 \%$ of the screw sites demonstrated some osteoconductivity, with nearly complete or complete ossification present in $47 \%{ }^{20}$ Another study assessed a PLLA $(75 \%) / \mathrm{HA}$ $(25 \%)$ interference screw at intervals out to 5 years. ${ }^{21}$ Using MRI instead of CT scanning, the authors reported that at 2 years $89 \%$ of the femoral tunnels with the PLLA $(75 \%) / H A(25 \%)$ screw showed either type 2 or 3 ossification. However, by 5 years this ossification had advanced to type 3 or 4 in $88 \% .^{21}$
Karikis et al. ${ }^{22}$ reported that $83 \%$ of patients 5 years after hamstring ACL reconstructions secured with selfreinforced SR-PL (96)/D (4) LA/ $\beta$-TCP biocomposite interference screws had decreased tibial tunnel widths compared with the early postoperative period. As Pinczewski and Salmon ${ }^{23}$ noted, biocomposite material reabsorption also may be influenced by the environment into which it is implanted, with the potential for different performance in different locations.

The PL (96)/D (4) LA/ $\beta$-TCP interference fixation screw used in the current study demonstrated osteoconductive behavior, was comparable with other successful biocomposite materials, and was associated with no adverse events. No cystic changes or tunnel widening were observed in the bone-patella tendon-bone ACL reconstruction model, and type 3 and type 4 osteoconductivity was observed in $25 \%$ of the screw sites.

\section{Limitations}

This study lacks a contemporaneous control group, relying on published historical controls. The Barber-Dockery ossification score, which at this point is the only available and published method, may be examiner-dependent and is a semiquantitative, subjective assessment of the screw site and the surrounding bony structures. No biopsy material was available to confirm the nature of what was present at the screw sites. The sample size was limited to 20 patients because of ethical concerns, and not all patients undergoing ACL reconstruction during the interval were subjected to CT scanning.

\section{Conclusions}

The PL (96)/D (4) LA/ $\beta$-TCP interference screw was replaced by calcified, nontrabecular material a mean of 42 months after implantation in a bone-patellar tendon-bone ACL reconstruction model. Osteoconductivity was confirmed.

\section{Acknowledgments}

The authors thank Kathy Berry for her assistance with this project.

\section{References}

1. Barber FA, Elrod BF, McGuire DA, Paulos LE. Preliminary results of an absorbable interference screw. Arthroscopy 1995; 1 1:537-548.

2. Barth J, Akritopoulos P, Graveleau N, et al. Efficacy of osteoconductive ceramics in bioresorbable screws for anterior cruciate ligament reconstruction: A prospective intrapatient comparative study. Orthop J Sports Med 2016:4:2325967116647724.

3. Tibor L, Chan PH, Funahashi TT, et al. Surgical technique trends in primary ACL reconstruction from 2007 to 2014. J Bone Joint Surg Am 2016;98:1079-1089. 
4. Barber FA, Dockery WD. Long-term absorption of betatricalcium phosphate poly-L-lactic acid interference screws. Arthroscopy 2008;24:441-447.

5. Barber FA, Dockery WD, Hrnack SA. Long-term degradation of a poly-lactide co-glycolide/beta-tricalcium phosphate biocomposite interference screw. Arthroscopy $2011 ; 27: 637-643$.

6. Barber FA, Dockery WD, Cowden CH 3rd. The degradation outcome of biocomposite suture anchors made from poly L-lactide-co-glycolide and beta-tricalcium phosphate. Arthroscopy 2013;29:1834-1839.

7. Ntagiopoulos PG, Demey G, Tavernier T, Dejour D. Comparison of resorption and remodeling of bioabsorbable interference screws in anterior cruciate ligament reconstruction. Int Orthop 2015:39:697-706.

8. Barber FA, Dockery WD. Long-term degradation of selfreinforced poly-levo (96\%)/dextro (4\%)-lactide/beta-tricalcium phosphate biocomposite interference screws. Arthroscopy 2016;32:608-614.

9. Barber FA, Dockery WD. Long-term absorption of poly-Llactic acid interference screws. Arthroscopy 2006;22:820-826.

10. Barber FA, Spenciner DB, Bhattacharyya S, Miller LE. Biocomposite implants composed of poly(lactide-coglycolide)/beta-tricalcium phosphate: Systematic review of imaging, complication, and performance outcomes. Arthroscopy 2017;33:683-689.

11. Konan S, Haddad FS. The unpredictable material properties of bioabsorbable PLC interference screws and their adverse effects in ACL reconstruction surgery. Knee Surg Sports Traumatol Arthrosc 2009;17:293-297.

12. Cox CL, Homlar KC, Carey JL, Spindler KP. CALAXO osteoconductive interference screw: The value of postmarket surveillance. J Surg Orthop Adv 2010;19:121-124.

13. Alonso B, Sobron FB, Vidal C, Vaquero J. Pretibial pseudocyst after anterior cruciate ligament reconstruction with a biocomposite screw. Acta Ortop Mex 2016;30:150-153 [in Spanish].

14. Spruit M, Meijers H, Obradov M, Anderson PG. CT density measurement of bone graft within an intervertebral lumbar cage: Increase of hounsfield units as an indicator for increasing bone mineral content. J Spinal Disord Tech $2004 ; 17: 232-235$.
15. Tashman S, Anderst W. In-vivo measurement of dynamic joint motion using high speed biplane radiography and CT: Application to canine ACL deficiency. J Biomech Eng 2003;125:238-245.

16. Webster KE, Feller JA, Elliott J, Hutchison A, Payne R. A comparison of bone tunnel measurements made using computed tomography and digital plain radiography after anterior cruciate ligament reconstruction. Arthroscopy 2004;20:946-950.

17. Shelbourne KD, Barnes AF, Gray T. Correlation of a single assessment numeric evaluation (SANE) rating with modified Cincinnati knee rating system and IKDC subjective total scores for patients after ACL reconstruction or knee arthroscopy. Am J Sports Med 2012;40: 2487-2491.

18. Storey TF, Montgomery WJ, Bush CH, Moser M. Magnetic resonance imaging appearance of anterior cruciate ligament reconstruction using Calaxo screws. Skeletal Radiol 2011;40:229-232.

19. Bourke HE, Salmon LJ, Waller A, et al. Randomized controlled trial of osteoconductive fixation screws for anterior cruciate ligament reconstruction: A comparison of the Calaxo and Milagro screws. Arthroscopy 2013;29: 74-82.

20. Johnston M, Morse A, Arrington J, Pliner M, Gasser S. Resorption and remodeling of hydroxyapatite-poly-Llactic acid composite anterior cruciate ligament interference screws. Arthroscopy 2011;27:1671-1678.

21. Arama Y, Salmon LJ, Sri-Ram K, et al. Bioabsorbable versus titanium screws in anterior cruciate ligament reconstruction using hamstring autograft: A prospective, blinded, randomized controlled trial with 5-year followup. Am J Sports Med 2015;43:1893-1901.

22. Karikis I, Ejerhed L, Sernert N, Rostgard-Christensen L, Kartus J. Radiographic tibial tunnel assessment after anterior cruciate ligament reconstruction using hamstring tendon autografts and biocomposite screws: A prospective study with 5-year follow-up. Arthroscopy 2017;33: 2184-2194.

23. Pinczewski LA, Salmon LJ. Editorial Commentary: The acrid bioscrew in anterior cruciate ligament reconstruction of the knee. Arthroscopy 2017;33:2195-2197. 